\title{
A Comparison of Bending Properties for Cellular Core Sandwich Panels
}

\author{
Li Yang ${ }^{1}$, Ola Harrysson ${ }^{2}$, Harvey West ${ }^{2}$, Denis Cormier ${ }^{3}$ \\ ${ }^{1}$ Department of Industrial Engineering, University of Louisville, Louisville, USA; ${ }^{2}$ Department of Industrial \& Systems Engineering, \\ North Carolina State University, Raleigh, USA; ${ }^{3}$ Department of Industrial \& Systems Engineering, Rochester Institute of Technology, \\ Rochester, USA. \\ Email: lyang5@ncsu.edu
}

Received May $15^{\text {th }}, 2013$; revised June $21^{\text {st }}, 2013$; accepted July $2^{\text {nd }}, 2013$

Copyright (C) 2013 Li Yang et al. This is an open access article distributed under the Creative Commons Attribution License, which permits unrestricted use, distribution, and reproduction in any medium, provided the original work is properly cited.

\begin{abstract}
In this study, various sandwich panel structures with different reticulate lattice core geometries were designed and then fabricated in titanium via the electron beam melting (EBM) process. Bending tests were performed on the titanium samples, and mechanical properties such as modulus, bending strength, and energy absorption were evaluated. Different failure mechanisms were observed, and it was found that sandwich structures with auxetic cores exhibited more homogeneous deflection and bending compliance compared with other structures. It was also demonstrated that properties of auxetic sandwich structures can be tailored using different cell structure geometries to suit the needs of a given design application. Furthermore, it was found that other 3D cellular sandwich structures can also exhibit high stiffness and strength, which is desirable in potential applications.
\end{abstract}

Keywords: Auxetic Structure; Cellular Structure; Sandwich Panels; Electron Beam Melting; Bending Properties

\section{Introduction}

3D cellular structures possess unique advantages with respect to specific strength, specific modulus, and energy absorption at low densities. They are therefore promising for applications that require light weight (e.g. aerospace, automotive, etc.). Two-dimensional cellular structures and foams are frequently used as sandwich structure cores in order to provide increased bending and shearing stiffness as well as energy absorption ability [1].

Among the range of possible cellular geometries, auxetic structures are of specific interest to researchers. Auxetic structures exhibit negative Poisson's ratio in one or more directions. These structures have been shown to exhibit significantly improved shear performance compared with regular structures [2-4]. According to the theory of elasticity, the shear modulus of negative Poisson's ratio structures could become even larger than the bulk moduli, making the structures ideal for use in sandwich panel cores [5-7]. Furthermore, auxetic structures exhibit synclastic bending [8-9], which also favors their potential applications in curved sandwich panels and sandwich skins for various applications.

Traditionally, auxetic structures have been fabricated through multi-step processes in which specific control over the cell geometry (i.e. strut sizes and angles) is quite difficult. The relatively recent emergence of polymer and metal additive manufacturing processes has given engineers the ability to fabricate parts with precise cellular geometries directly from the CAD models. It therefore has become possible to fabricate these structures and to compare their experimentally determined material properties with predicted values.

In the current study, sandwich structures with re-entrant auxetic cores as well as several other $3 \mathrm{D}$ reticulate cellular core geometries were designed in CAD and then fabricated via Arcam's electron beam melting (EBM) process. Mechanical properties pertinent to sandwich structure performance were measured, including stiffness, strength and energy absorption. A comparison of the bending properties between different auxetic core designs is provided to evaluate their potential in structural applications.

\section{Structural Designs}

The unit cell of the 3D re-entrant lattice auxetic structure used in this study is shown in Figure 1(a) $[10,11]$. This auxetic structure is an orthotropic structure with directions $x$ and $y$ exhibiting identical properties due to the 


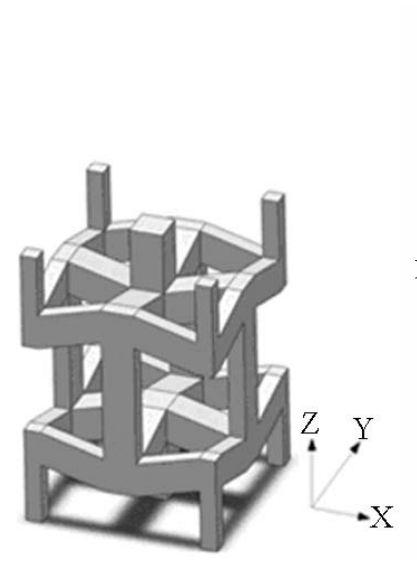

(a)

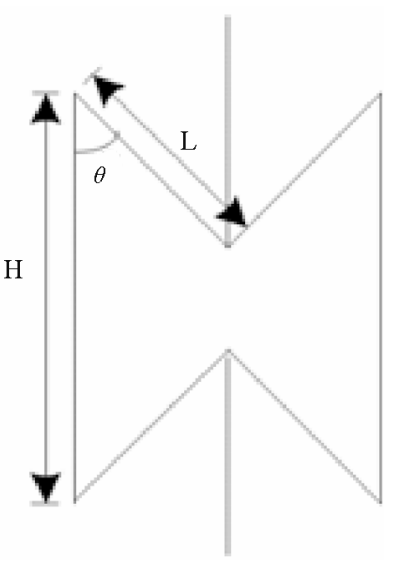

(b)
Figure 1. Design of the 3D re-entrant lattice structure.

symmetry. Therefore, the design of the structure could be represented by the simplified $2 \mathrm{D}$ geometry shown in Figure 1(b), which includes the length of the vertical $(H)$ and re-entrant $(L)$ struts, the re-entrant angle $\theta$, and the thickness $(t)$ of the strut (not shown in the figure). Li et al. [12] showed that compressive properties of this auxetic structure could be estimated as a function of design parameters $H, L, \theta$, and $t$. The relationship between the Poisson's ratio values and the design parameters could be written as:

$$
\begin{aligned}
& v_{z}=-\frac{\left(\frac{L^{2}}{E t_{1} t_{2}^{3}}+\frac{6}{5 G t_{1} t_{2}}\right) \cos \theta(\alpha-\cos \theta)}{\frac{L^{2} \sin ^{2} \theta}{E t_{1} t_{2}^{3}}+\frac{6 \sin ^{2} \theta}{5 G t_{1} t_{2}}+\frac{4 \alpha}{E t_{3} t_{4}}} \\
& v_{x}=-\frac{\sin ^{2} \theta}{\cos \theta(\alpha-\cos \theta)}
\end{aligned}
$$

where $v_{z}$ and $v_{x}$ are Poisson's ratios of the structure under compression in the $z$ and $x$ directions respectively. Poisson's ratios along the $x$ and $y$ axes are identical due to symmetry of the structure (i.e. $v_{x}=v_{y}$ ). $E$ and $G$ are Young's modulus and shear modulus, $\alpha=H / L, t_{1}$ and $t_{2}$ are dimensions of the re-entrant struts, and $t_{3}$ and $t_{4}$ are dimensions of the vertical struts. The cross section of the struts is taken as rectangular for this analysis, although similar analysis' can be performed for other cross sections.

The Poisson's ratio values are expected to have a significant effect on the mechanical properties of the re-entrant auxetic structures. With greater negative Poisson's ratio values, the modulus and strength of the structures will also become greater $[11,12]$. In the current study, two configurations were designed for the re-entrant auxetic structure with significantly different Poisson's ratios, as shown in Table 1. In Table 1, $t_{V}$ and $t_{R}$ stand for the thickness of the square shaped vertical struts and reentrant struts, respectively.

The sandwich panels for bending tests were designed as illustrated in Figure 2 using the two different auxeticcore designs $\left(A_{1}\right.$ and $\left.A_{2}\right)$. The dimensions of the sandwich panel cores were kept at approximately $17 \mathrm{~mm} \times 20$ $\mathrm{mm} \times 150 \mathrm{~mm}$ while maintaining structural symmetry in each direction. In addition, the thickness of the sandwich skins was fixed at $0.75 \mathrm{~mm}$. The resulting structures had $1 \times$ $2 \times 14$ auxetic unit cell repetitions for design $\mathrm{A}_{1}$, and $1.5 \times$ $2 \times 20$ auxetic unit cell repetitions for design $A_{2}$.

From the unit cell orientation as shown in Figure 2, it was known that under a bending load, the auxetic structures would be subject to compressive stress normal to the bending load direction. According to Equation (2), design $\mathrm{A}_{2}$ will have a higher $v_{x}$ value and would therefore be expected to exhibit higher strength and higher modulus than design $\mathrm{A}_{1}$.

In order to compare the auxetic geometries with other reticulate cellular geometries, octahedral, rhombic, and hexagonal cellular structures were designed in CAD. These geometries have been demonstrated elsewhere and fabricated by electron beam melting [13-15]. The unit cells of these structures are shown in Figure 3. Parameter values for each structure are shown in Table 2. The resulting sandwich panels had unit cell array counts of $2 \times$ $2 \times 19$ for the octahedral panel, $2 \times 2 \times 18$ for the rhombic panel, and $3 \times 3 \times 28$ for the hexagonal panel. The sandwich skin thickness for each design was fixed at 1 $\mathrm{mm}$.

All of the cellular structure designs except for $\mathrm{A}_{1}$ possess similar relative densities as shown in Tables $\mathbf{1}$ and $\mathbf{2}$. According to the cellular theory, the relative density of a structure has a significant influence on its mechanical properties [16]. The selected geometries therefore permit a high level comparison of material properties between the different cellular geometries.

\section{Experimental Procedures}

Three titanium (Ti-6Al-4V) samples for each sandwich panel design were fabricated using an Arcam A2 EBM system using $+325 /-100$ spherical powder. The powder was made via the plasma rotating electrode (PREP) process. Identical default process settings for electron

Table 1. Design parameter values for the re-entrant auxetic structure.

\begin{tabular}{ccccccccc}
\hline Design & $H(\mathrm{~mm})$ & $L(\mathrm{~mm})$ & $\theta($ Deg. $)$ & $t_{V}(\mathrm{~mm})$ & $t_{R}(\mathrm{~mm})$ & $v_{x}$ & $v_{z}$ & \multicolumn{2}{c}{ Relative Density $(\%)$} \\
\hline $\mathrm{A}_{1}$ & 15 & 7.5 & 45 & 1 & 0.707 & -1.704 & -0.547 & 6.3 \\
$\mathrm{~A}_{2}$ & 7.595 & 4 & 70 & 1 & 0.940 & -0.445 & -1.658 & 11.6 \\
\hline
\end{tabular}




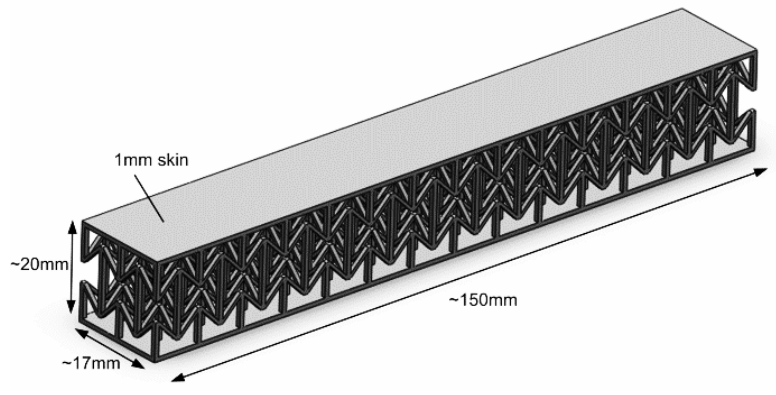

Figure 2. The sandwich panel with auxetic core.

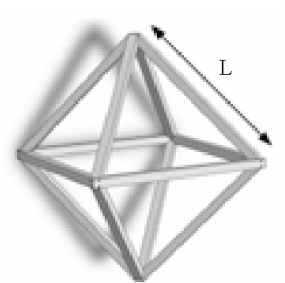

(a)

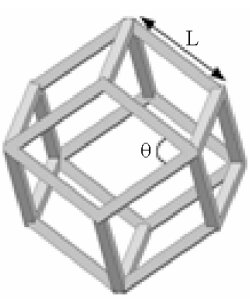

(b)

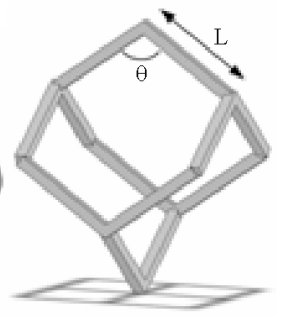

(c)
Figure 3. Unit cell designs for (a) octahedral; (b) rhombic; and (c) hexagonal structures.

Table 2. Designs of various cellular structures.

\begin{tabular}{ccccc}
\hline Design & $L(\mathrm{~mm})$ & $\theta$ (Deg.) & $t(\mathrm{~mm})$ & $\begin{array}{c}\text { Relative } \\
\text { Density }(\%)\end{array}$ \\
\hline O (Octahedral) & 8 & - & 1 & 11.98 \\
R (Rhombic) & 4.65 & 60 & 1 & 11.00 \\
H (Hexagonal) & 3.19 & 120 & 1 & 11.72 \\
\hline
\end{tabular}

beam melting of lattice geometries were used to produce all samples. All samples were oriented in the build chamber such that the two face skins were normal to the build direction. Due to build chamber size limitations, the 18 samples (total) were fabricated in two batches. After the samples were cleaned, their dimensions were measured using digital calipers, and their masses were measured using a digital balance having a resolution of $0.0001 \mathrm{~g}$.

Bending tests were carried out using an Applied Test System $1620 \mathrm{C}$ at a constant strain rate of $1.27 \mathrm{~mm} / \mathrm{min}$. Three point bending tests were carried out with a support span of $L=114.3 \mathrm{~mm}$ as shown in Figure 4. The support rollers had a diameter of $12.7 \mathrm{~mm}$, and the load roller had a diameter of $25.4 \mathrm{~mm}$. The displacement and load $(F)$ of the loading roller were recorded through the crosshead. The test was automatically stopped when the loading level of the roller dropped below $70 \%$ of the maximum recorded loading level. The actual experimental setup is further illustrated in Figure 5. An FEA analysis for each model was also performed in order to compare predicted material properties with actual measured properties. As shown in Figure 6, each model was fixed at two strips on

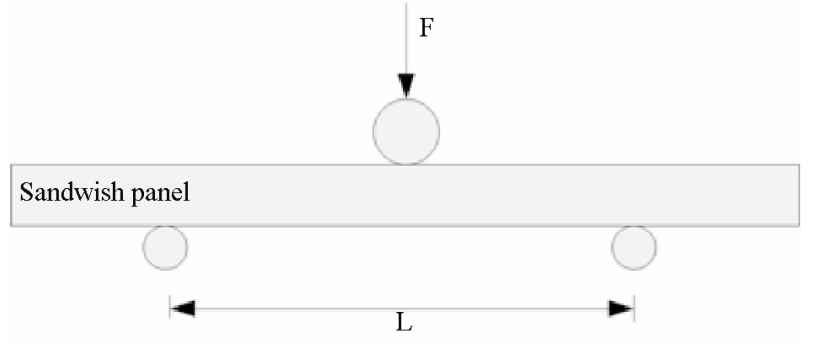

Figure 4. Experimental setup for the three point bending test.

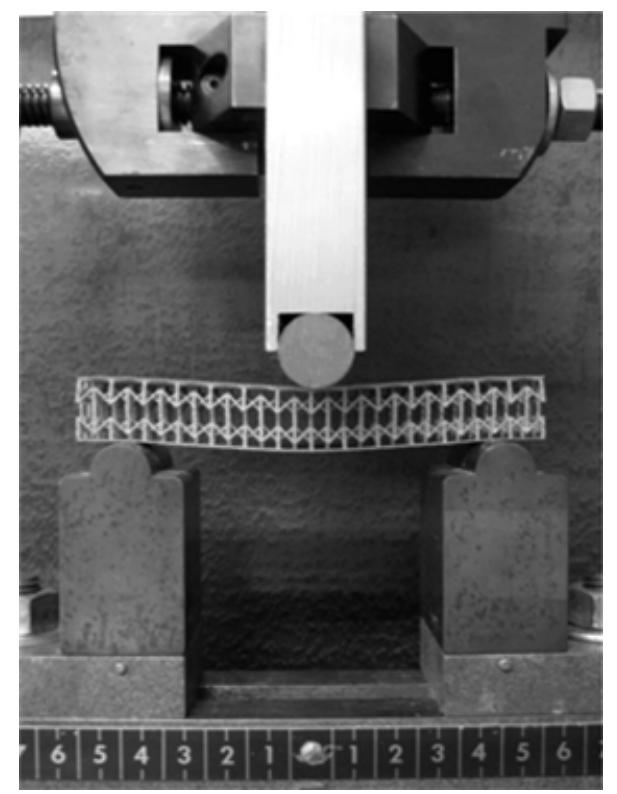

Figure 5. Actual setup for the bending test.

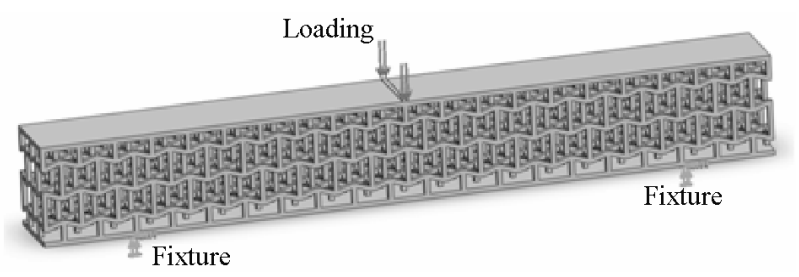

Figure 6. FEA of the bending of the sandwich panel.

the bottom face, and then loaded at the strip on the center of the top face. The width of each strip was $1 \mathrm{~mm}$. During the FEA studies, loadings ranging from 1000 - 5000 $N$ were simulated for each structure. The strength values were obtained by determining the minimum loading level for which the stress across the entire cross section of any strut exceeded the yield strength of Ti-6Al-4V (1050 $\mathrm{MPa})$.

\section{Results and Discussion}

The measured dimensions of fabricated titanium samples are listed in Table 3. In Table 3,D1 and D2 are the 
Table 3. Actual parameters of the samples made by EBM.

\begin{tabular}{cccccc}
\hline Design & $D 1(\mathrm{~mm})$ & $D 2(\mathrm{~mm})$ & $L(\mathrm{~mm})$ & Mass $(\mathrm{g})$ & Relative Density $(\%)$ \\
\hline $\mathrm{A}_{1}$ & $20.405 \pm 0.259$ & $22.538 \pm 0.125$ & $148.675 \pm 0.155$ & $45.063 \pm 2.688$ & $14.87 \pm 0.81$ \\
$\mathrm{~A}_{2}$ & $19.829 \pm 0.153$ & $16.222 \pm 0.029$ & $150.334 \pm 0.140$ & $45.852 \pm 1.504$ & $21.40 \pm 0.60$ \\
$\mathrm{O}$ & $23.292 \pm 0.116$ & $17.052 \pm 0.029$ & $151.875 \pm 0.125$ & $48.646 \pm 0.616$ & $18.20 \pm 0.22$ \\
$\mathrm{R}$ & $19.668 \pm 0.205$ & $17.230 \pm 0.039$ & $144.848 \pm 0.073$ & $41.647 \pm 1.965$ & $19.15 \pm 0.73$ \\
$\mathrm{H}$ & $20.616 \pm 0.106$ & $17.729 \pm 0.000$ & $160.274 \pm 0.076$ & $52.112 \pm 1.242$ & $20.08 \pm 0.38$ \\
\hline
\end{tabular}

overall thickness and width of the sandwich panels, and $L$ is the total length. It could be seen that the dimensions of the samples were quite consistent, indicating stable process quality. The relative densities of the samples were significantly larger than 0.1 due to the existence of the surface skins. All the designs, except for $A_{1}$, had measured relative densities close to 0.2 .

The thickness of the sandwich skins for auxetic designs $A_{1}$ and $A_{2}$ were around $0.75 \mathrm{~mm}$, while the thickness of the skins for the other sandwich structures were very close to $1 \mathrm{~mm}$. According to the classic theory of sandwich panels, skin thickness is the dominant factor for the performance of the structure. However, preliminary FEA results with the designed structures showed that the skin thickness hada minimal effect on the bending modulus of the sandwich panels. It is hypothesized that this is due to the very low relative density of the core structure which leads to large compliance that accommodates local deflections of the two skin panels. Furthermore, preliminary study showed that for auxetic sandwich structures, the dominant failure mode during bending was core shear, which is in turn determined by the geometries of individual struts rather than the surface skin.

During bending, the upper region of the sandwich panel is subject to compressive stress, while the lower region is subject to tensile stress. For regular core structures, compressive stresses result in lateral expansion. As a consequence, localized stress concentrations and potential wrinkling on the face skins can be expected. Conversely, auxetic structures with negative Poisson's ratios exhibit lateral shrinkage (or inward movement) under compression and will therefore better accommodate macroscopic structural deformation.

Figure 7(a) shows the auxetic sandwich panel deformation during testing, whereas Figure 7(b) shows the corresponding FEA simulation result. Figures 7(a) and (b) both show very little localized deformation during bending. Instead, the deflection is distributed along the length of the sandwich panel homogeneously. Whereas sandwich panels with foam cores typically fail via face yield, core shear, indentation, delamination and face wrinkle [5], the auxetic sandwich panels failed by fracture of vertical struts under the combination of bending and tension. Failure occurred at the vertical struts located roughly at the middle section between the loading roller and the support roller where the local deformation was at its maximum as seen in Figure 7(a). Because of the highly homogeneous distribution of deflection and stress, this auxetic sandwich structure could potentially be useful for applications such as structural beams. Since the vertical struts are subject to critical failure, new designs involving non-uniform strut sizes could be applied to further improve the structural performance.

Figure 8 shows the deflection of the other sandwich structures in FEA simulation. Again, the tested titanium structures exhibited similar behavior as that predicted from the FEA studies. It is apparent that for octahedral, rhombic and hexagonal sandwiches, the stress distributions, and therefore the deformation of the structures, was largely concentrated at the surface area where the structure was loaded. Upon failure, face yield was observed for all three of these geometries.

Table 4 shows the bending strength and modulus results, as well as the total energy absorption for each type of structure. The results for rhombic and octahedral samples showed an unusually high variation in maximum force and therefore, the strength and the total energy absorption. It is noted that in order to produce comparable relative densities between sample geometries, the rhombic and octahedral samples had fewer unit cell repetitions through the thickness of the samples. It is surmised that the small number of unit cell repetitions through the thickness of the samples contributed to this elevated degree of variance. All the other type of structures showed relatively consistent properties.

Comparing $A_{1}$ and $A_{2}$, it is apparent that $A_{2}$ exhibited significantly higher strength and modulus, as predicted. With higher $v_{z}$ value, the modulus of $\mathrm{A}_{2}$ is about 7 times that of $A_{1}$, while the strength of $A_{2}$ had an approximately $200 \%$ increase compared with $A_{1}$. On the other hand, design $A_{1}$ exhibited significantly higher resilience compared with $A_{2}$. The maximum deflection for $A_{1}$ was almost twice as much as that of $A_{2}$. Although design $A_{1}$ could withstand much greater deflection than $\mathrm{A}_{2}$, design $\mathrm{A}_{2}$ absorbed a considerable amount of energy during the bending, as also shown in Table 4.

The octahedral, rhombic, and hexagonal sandwich panels showed relatively high strength and modulus val- 


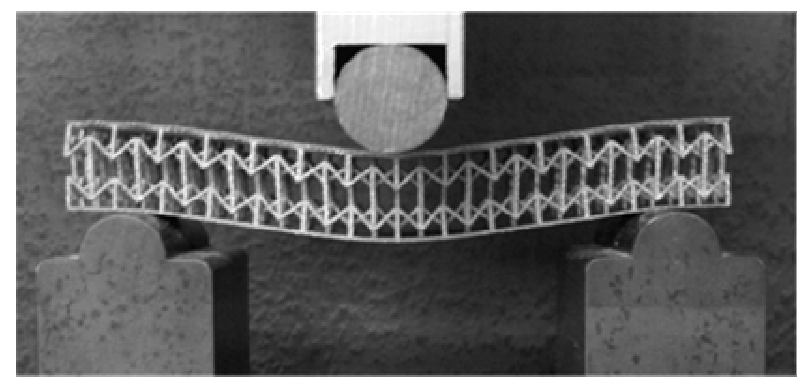

(a)

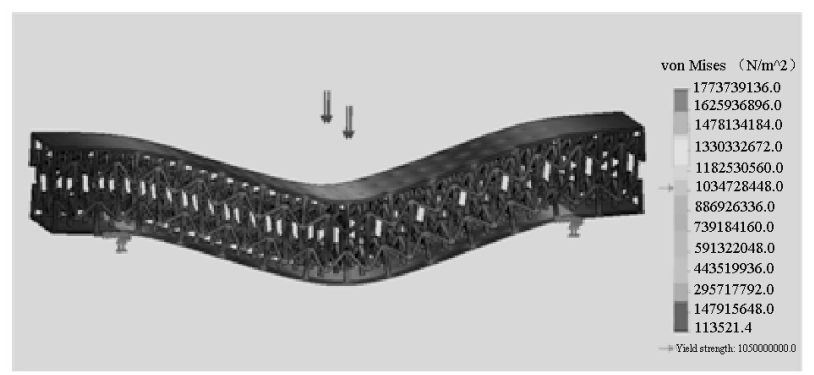

(b)

Figure 7. The shape of (a) titanium auxetic sandwich and (b) FEA model under bending.

ues compared with the auxetic sandwiches. However, the maximum deflections of these structures were significantly lower, indicated that these structures had rather low overall structural ductility.

From the comparison, it was apparent that the auxetic structures showed significantly superior performance in terms of maximum deflection that can be tolerated. Although design $A_{1}$ exhibited lower strength and modulus compared to the other designs, it absorbed a significant amount of energy. Design $\mathrm{A}_{2}$ showed higher modulus and strength compared to $A_{1}$. Furthermore, the total energy absorption of $A_{2}$ was about $100 \%$ higher than that of $\mathrm{A}_{1}$. The octahedral, rhombic and hexagonal structures showed significantly lower ductility compared to the auxetic sandwich structures, while possessing higher modulus and potentially strength values. As a result, these structures could exhibit energy absorption abilities comparable to the auxetic sandwich with larger negative Poisson's ratio values in the thickness direction.

It is known that for many applications, the energy absorption of the sandwich panels during bending is of great interest. For energy absorption purposes, it is desired that the structure exhibit low peak response force, and high total energy absorption. Comparing the structures in Table 4, it is apparent that the auxetic structures possess significant advantages over the other unit cell geometries. At a similar peak response force level (around $3000 \mathrm{~N}$ ), the auxetic sandwich designs absorbed about $100 \%$ more energy than the other designs. At the same energy absorption level (about $9 \mathrm{~J}$ ), the auxetic structures had a response force of about $1200 \mathrm{~N}$, which

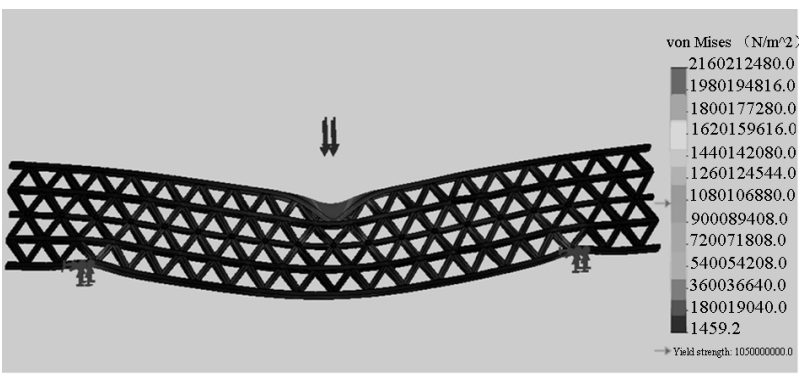

(a)

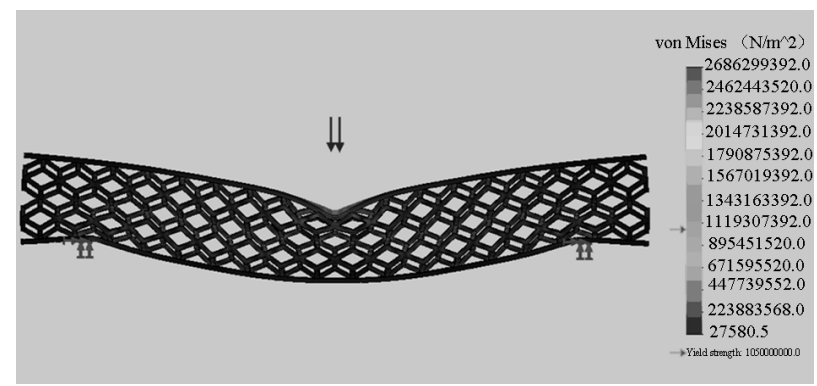

(b)

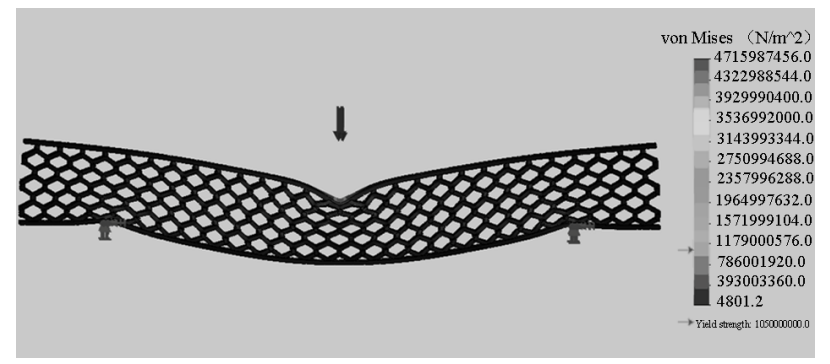

(c)

Figure 8. FEA of the bending of various sandwich structures. (a) Octahedral; (b) Rhombic; (c) Hexagonal.

was significantly lower than the others. The other structures do not seem to be ideal candidates for energy absorption applications due to their significantly higher response force level. However, their higher modulus and strength properties make them potential candidates for applications where high specific stiffness and static strength of the sandwich panels are required.

A comparison between the FEA and the average experimental results is shown in Table 5. The strength values of the FEA agree quite well with the experimental results, while the modulus values from the FEA study are uniformly higher than experimental results, especially for the octahedral and hexagonal structures. Given that the crosshead displacement was used to monitor the deflection of the beam, the compliance of the load cell, when testing stiffer structures would reduce the measured modulus.

It is worth noting that the ability of additive manufacturing processes such as EBM to fabricate engineered cellular structures with any desired strut sizes and angles opens up tremendous possibilities for further design op- 
Table 4. Bending properties of various structures.

\begin{tabular}{cccccc}
\hline Design & Max. Force $(\mathrm{N})$ & Max. Deflection $(\mathrm{mm})$ & Strength $(\mathrm{MPa})$ & Modulus $(\mathrm{GPa})$ & Energy Abs. $(\mathrm{J})$ \\
\hline $\mathrm{A}_{1}$ & $1206.43 \pm 37.35$ & $9.20 \pm 0.77$ & $22.04 \pm 0.21$ & $0.39 \pm 0.02$ & $8.73 \pm 0.50$ \\
$\mathrm{~A}_{2}$ & $3432.23 \pm 58.58$ & $5.96 \pm 0.27$ & $92.26 \pm 1.62$ & $2.98 \pm 0.04$ & $16.54 \pm 1.61$ \\
$\mathrm{O}$ & $5714.50 \pm 977.40$ & $2.61 \pm 0.48$ & $105.99 \pm 18.83$ & $5.23 \pm 0.78$ & $9.08 \pm 0.17$ \\
$\mathrm{R}$ & $3163.86 \pm 966.96$ & $2.92 \pm 0.76$ & $81.06 \pm 23.39$ & $4.65 \pm 0.51$ & $6.21 \pm 4.39$ \\
$\mathrm{H}$ & $5261.75 \pm 199.04$ & $3.13 \pm 0.26$ & $119.70 \pm 3.48$ & $6.20 \pm 0.45$ & $16.78 \pm 2.68$ \\
\hline
\end{tabular}

Table 5. Comparison of FEA and experiments.

\begin{tabular}{ccccc}
\hline Design & Measured Strength $(\mathrm{MPa})$ & FEA Strength $(\mathrm{MPa})$ & Measured Modulus $(\mathrm{GPa})$ & FEA Modulus $(\mathrm{GPa})$ \\
\hline $\mathrm{A}_{1}$ & 22.04 & 26.56 & 0.39 & 0.47 \\
$\mathrm{~A}_{2}$ & 92.26 & 75.00 & 2.98 & 3.26 \\
$\mathrm{O}$ & 105.99 & 103.12 & 5.23 & 10.85 \\
$\mathrm{R}$ & 81.06 & 82.50 & 4.65 & 6.46 \\
$\mathrm{H}$ & 119.70 & 111.94 & 6.20 & 9.06 \\
\hline
\end{tabular}

timization of all of the cellular geometries examined in this paper. For instance, critically loaded struts can be fabricated with slightly larger diameters than other struts.

\section{Conclusions}

In the current work, sandwich panels with different cellular cores were designed and compared. Although the 3D reticulate cellular core structures were not optimized for bending, their overall performance showed promising potential as future sandwich cores. During bending, the auxetic sandwich panels exhibited homogeneous distribution of stress and deformation. Failure by fracture of the vertical struts located roughly at the middle section between the loading and support rollers was seen in all cases. Future studies should therefore focus on optimization of the cellular structure based on the expected loading patterns. Thickening of the critical vertical struts would potentially lead to significant enhancements in material properties with relatively little increase in mass. The other sandwich structures showed significant stress concentration at the loading area, and failed by face yield.

The bending tests revealed that with different Poisson's ratio values, the mechanical properties of the auxetic sandwich panels could be tailored over a wide range. In addition, the auxetic sandwich panels also exhibited extraordinary resilience under bending compared with all the other structures. As a result, the auxetic sandwich panels generally possess considerable potential specifically for energy absorption applications.

While the non-auxetic cellular geometries studied here had relatively low ductility and arbitrarily chosen geometrical parameters, they exhibited relatively high strength and modulus. In addition, some of these designs also exhibited high energy absorption during bending due to the high modulus. In conclusion, these structures have potential in applications where stiff and strong structural beams are employed, and where high toughness is needed.

\section{REFERENCES}

[1] H. G. Allen, "Analysis and Design of Structural Sandwich Panels," Pergamon Press, Oxford, 1969.

[2] R. Lakes, "Foam Structures with a Negative Poisson's Ratio," Science, Vol. 235, No. 4792, 1987, pp. 1038-1040. doi:10.1126/science.235.4792.1038

[3] F. Scarpa and P. J. Tomlin, "On the Transverse Shear Modulus of Negative Poisson's Ratio Lattice Structures," Fatigue \& Fracture of Engineering Materials \& Structures, Vol. 23, No. 8, 2000, pp. 717-720. doi:10.1046/j.1460-2695.2000.00278.x

[4] F. Scarpa and G. Tomlinson, "Theoretical Characteristics of the Vibration of Sandwich Plates with In-Plane Negative Poisson's Ratio Values," Journal of Sound and Vibration, Vol. 230, No. 1, 2000, pp. 45-67. doi:10.1006/jsvi.1999.2600

[5] T. M. McCormack, R. Miller, O. Kesler and L. J. Gibson, "Failure of Sandwich Beams with Metallic Foam Cores," International Journal of Solids and Structures, Vol. 38, No. 28, 2001, pp. 4901-4920. doi:10.1016/S0020-7683(00)00327-9

[6] O. Kesler and L. J. Gibson, "Size Effects in Metallic Foam Core Sandwich Beams," Materials Science and Engineering: A, Vol. 326, No. 2, pp. 228-234. doi:10.1016/S0921-5093(01)01487-3

[7] C. Chen, A.-M. Harte and N. A. Fleck, "The Plastic Collapse of Sandwich Beams with a Metallic Foam Core," International Journal of Mechanical Sciences, Vol. 43, No. 6, 2001, pp. 1483-1506.

[8] K. E. Evans, "The Design of Doubly Curved Sandwich Panels with Honeycomb Cores," Composite Structures, Vol. 17, No. 2, 1991, pp. 95-111. 
[9] R. Lakes, "Advances in Negative Poisson's Ratio Materials," Advanced Materials, Vol. 5, No. 4, 1993, pp. 293296. doi:10.1002/adma.19930050416

[10] L. Yang, D. Cormier, H. West and K. Knowlson, "NonStochastic Ti-6Al-4V Foam Structure That Shows Negative Poisson's Ratios," Materials Science and Engineering: $A$, Vol. 558, 2012, pp. 579-585. doi:10.1016/j.msea.2012.08.053

[11] L. Yang, O. Harrysson, D. Cormier and H. West, "Compressive Properties of Ti-6Al-4V Auxetic Mesh Structures Made by EBM Process," Acta Materialia, Vol. 60, No. 8, 2012, pp. 3370-3379.

doi:10.1016/j.actamat.2012.03.015

[12] L. Yang, O. Harrysson, D. Cormier and H. West, "Modeling of the Uniaxial Compression of a 3D Periodic ReEntrant Honeycomb Structure," Journal of Materials Science, Vol. 48, No. 4, 2012, pp. 1413-1422.

doi:10.1007/s10853-012-6892-2
[13] O. L. A. Harrysson, O. Cansizoglu, D. J. Marcellin-Little, D. Cormier and H. West II, "Direct Metal Fabrication of Titanium Implants with Tailored Materials and Mechanical Properties Using Electron Beam Melting Technology," Materials Science and Engineering: $C$, Vol. 28, No. 3, 2008, pp. 366-373. doi:10.1016/j.msec.2007.04.022

[14] O. Cansizoglu, O, Harrysson, D. Cormier, H. West and T. Mahale, "Properties of Ti-6Al-4V Non-Stochastic Lattice Structures Fabricated via Electron Beam Melting," Materials Science and Engineering: A, Vol. 492, No. 1-2, 2008, pp. 468-474.

[15] O. Cansizoglu, "Mesh Structures with Tailored Properties and Applications in Hip Stems," Ph.D. Dissertation, North Carolina State University, Raleigh, 2008.

[16] L. J. Gibson, "Cellular Solids: Structure and Properties," 2nd Edition, Cambridge University Press, New York, 1997. 